\title{
mi RNAS : In the Domain of Cancer Chemoresistance and Stem Cells
}

\section{Samdisha Dubey and Rachana Garg*}

Department of Biotechnology, National Institute of Pharmaceutical Education and

Research, Ahmedabad, Gujarat, India

*Corresponding Author: Rachana Garg, Department of Biotechnology, National Institute of Pharmaceutical Education and Research, Ahmedabad, Gujarat, India.

DOI: $10.31080 /$ ASCB.2020.04.0204
Received: December 30, 2019

Published: January 28, 2020

(C) All rights are reserved by Samdisha

Dubey and Rachana Garg.

\begin{abstract}
MicroRNAs are short non-coding RNAs that regulate the levels of proteins by acting on its target mRNA, thereby gaining a translational control. Certain cells have distinct levels of miRNA, therefore, any aberration in miRNA expression is directly proportional to the deregulation of the proteins which it regulates; this may in turn lead to a disease condition. Till date, several miRNAs have been reported to play role in different aspects of various diseases including cancer. The major difficulties being faced in the anti-cancer therapy are: chemoresistance and local reoccurrence due to cancer stem cells. Notably, miRNAs have found to be regulating pathways involved in chemoresistance and modulating cancer stem cell attributes. Here, we give a brief outline about the role of miRNAs in cancer chemoresistance and stem cells.
\end{abstract}

Keywords: Mirna; Chemoresistance; Cancer Stem Cells

MicroRNAs (miRNA) are small non-coding RNAs (ncRNAs) comprising of 20 to 24 nucleotides. They play essential role in the translational regulation of proteins chiefly by binding to the three major regions on the mRNA: 3' UTR, coding and 5' UTR. A single miRNA has the ability to regulate more than one protein. Till date, nearly 2000 miRNAs have been identified that are associated with regulation of human genome [1]. Besides, these miRNAs have also been identified in plants and other species, wherein they function similarly.

It has been two-decades since the identification of miRNAs and understanding of their biology. The group of Ambros and Ruvkun in 1993 reported the first mi RNA, lin 4 in C. Elegans [2], followed by the discovery of let- 7 in the year 2000 by Reinhart., et al [3]. Since then, there had been a growing interest amongst the researchers for not only the identification of new miRNAs but also in deciphering the mechanism underlying the translational regulation mediated by small non-coding RNAs. This has resulted in generating various in-silico tools and appli- cations that have significantly contributed in the determination of the miRNA targets [4].

Like any other RNA, synthesis of miRNA also begins with the key role of RNA polymerase II/III and is coded majorly by intragenic introns [5]. miRNA biogenesis has been classified into canonical and non-canonical pathways; canonical being the major driving force. The two pathway majorly differ in the source of gene from where the miRNA is transcribed. The common vital proteins and enzymes involved in these pathways are DiGeorge Syndrome Critical Region 8 (DGCR8), ribonuclease III enzyme-Drosha, exportin 5 (XPO5) / RanGTP complex, RNase III endonuclease Dicer and Argonaute (AGO) class of proteins. The genes are first transcribed in the nucleus into primary miRNA (pri-miRNA) and further processed into precursor miRNA (pre-miRNA) with the help of DGCR8 and Drosha.XP05/RanGTP complex aids in the translocation of the pre-miRNA to the cytoplasm where it is processed into a mature miRNA by the enzyme, 
Dicer. Matured miRNA in association with AGO proteins forms a RNA Induced Silencing Complex (RISC) that leads to the degradation of targeting mRNA. RISC- mediated silencing/degradation of mRNA is achieved majorly by deadenylation of target mRNA [5].

Notably, besides being involved in normal physiology, miRNAs have also been implicated in several diseases. miRNAs follow a distinct expression pattern in normal cells which however, in diseased condition is often disturbed and/or de-regulated. Aberrant miRNA expression affects the translation of various protein that are under its regulation, and this in turn, leads to a disease condition. Aberrant/Overexpression of miRNA has been reported in several cancer, viral, immunological and neuro-degenerative diseases [6]. Particularly, in cancer, a causal link has been ascribed to the deregulation of several miRNAs. Success in cancer treatment has majorly been halted due to the acquisition of chemoresistance and reoccurrence at a distant metastatic site, which could be a contribution from cancer stem cells. There are growing evidences indicating that miRNAs directly or indirectly governs both these crucial factors.

Talking about chemoresistance, it is majorly acquired due to continued administration of a particular drug. There are various key players and pathways that are involved in cancer chemoresistance and stem cells namely; NF- $\kappa \mathrm{B}, \mathrm{WNT} / \beta$-catenin, AKT-PI3Kinase, Notch signaling pathway, ABC transporter pumps: ABCG2, cancer stem cell markers (Nanog, Sox2, Oct4, Tcf3) and aldehyde dehydrogenase (ALDH) [7]. Various studies have indicated that the loss of tumor suppressive miRNAs increases the expression of oncogenes whilst increased expression of oncogenic miRNAs represses the tumor suppressor gene. This cumulative accumulation of oncogenes and depletion of tumor suppressive genes leads to differentiation as well as proliferation of cancer stem cells. The exponential growth of the cancer stem cells during any chemotherapy neutralises the effects to the drug being administered and ultimately results in chemoresistance against that drug. Doxorubicin, paclitaxel, platinum drugs are the drugs where majorly chemoresistance has been seen.

In lung cancer: miR221/222, miR30b/c, miR103, miR203 and miR34a have been implicated in the chemoresistance associated with various tyrosine kinase inhibitors. Likewise, miR-
505, miR-181a, miR-663, miR-25, miR-145, miR-664a, miR-128, miR-30c, miR-326, miR-181a and miR-34a have roles in doxorubicin resistance in breast cancer [8]. In gastric cancer: miR-15, miR-16, miR-21, miR-200bc/429, miR-181b, miR-497, miR-503, miR-143, miR-449, miR- 44ab, miR-508-5p, miR-223 and miR23-3p have proved their roles in the cisplatin chemoresistance [9]. These are just few of the many examples where miRNAs have been shown to be associated with the developing cancer chemoresistance. As highlighted above, miRNA dysregulation has also been linked with the functioning of cancer stem cells. For example, miR-191 [10] and miR-320 [11] through their modulation of $\mathrm{Wnt} / \beta$-catenin pathway have been associated with cancer stemness of lung and prostate cancer respectively. miR- 34a by acting on the Notch signalling pathway, plays a major role in breast cancer [12] and colon cancer stem cells [13]. Accordingly in recent years, miRNA have not only been used as a diagnostic marker but have also been employed as a therapeutic approach targeting cancer of various types.

Thus, developing miRNA-based therapy is receiving increasing attention and has become the one of the prime focus of the scientific research targeting cancer treatment. Many novel formulations are currently being designed for the delivery of miRNAs so as to maximize their bioavailability while minimizing the side effects, if any. Nano-formulations have proved to be the boon in the miRNA-based therapeutics system [14].

Overall, miRNAs serve as an important player in the regulation of many pathways in the carcinogenesis and offers potential in developing new therapeutic approach to combat cancer chemoresistance and the reoccurrence due to the self- renewal property of cancer stem cells.

\section{Bibliography}

1. Hammond SM. "An overview of microRNAs". Advanced Drug Delivery Reviews 87 (2015): 3-14.

2. Lee RC., et al. "The C. elegans heterochronic gene lin-4 encodes small RNAs with antisense complementarity to lin-14". Cell 75.5 (1993): 843-854.

3. Reinhart BJ., et al. "The 21-nucleotide let-7 RNA regulates developmental timing in Caenorhabditis elegans". Nature 403.6772 (2000): 901-906. 
4. Almeida MI., et al. "MicroRNA history: Discovery, recent applications, and next frontiers". Mutation Research - Fundamental and Molecular Mechanisms of Mutagenesis 717 (2011): 1-8.

5. O'Brien J., et al. "Overview of microRNA biogenesis, mechanisms of actions, and circulation". Frontiers in Endocrinology 9 (2018):1-12.

6. Li Y and Kowdley Kv. "MicroRNAs in Common Human Diseases. Vol. 10, Genomics, Proteomics and Bioinformatics". Beijing Genomics Institute (2012): 246-253.

7. Nurrul Abdullah L and Kai-Hua Chow E. Mechanisms of chemoresistance in cancer stem cells (2013).

8. Hu WZ., et al. "Functional miRNAs in breast cancer drug resistance". Onco Targets and Therapy Dove Medical Press Ltd 11 (2018): 1529-1541.

9. Wu WKK., et al. "MicroRNA dysregulation in gastric cancer: A new player enters the game". Oncogene 29 (2010): 5761-5771.

10. Xu W., et al. "MicroRNA-191, by promoting the EMT and increasing CSC-like properties, is involved in neoplastic and metastatic properties of transformed human bronchial epithelial cells". Molecular Carcinogenesis 54.S1 (2015): E148E161.

11. Hsieh IS., et al. "MicroRNA-320 suppresses the stem cell-like characteristics of prostate cancer cells by downregulating the Wnt/beta-catenin signaling pathway". Carcinogenesis 34.3 (2013): 530-538.

12. Kang L., et al. "MicroRNA-34a suppresses the breast cancer stem cell-like characteristics by downregulating Notch1 pathway". Cancer Science 106.6 (2015): 700-708.

13. Bu P., et al. "A microRNA miR-34a- regulated bimodal switch targets notch in colon cancer stem cells". Cell Stem Cell 12.5 (2013): 602-615.

14. Juliano R., et al. "Biological Barriers to Therapy with Antisense and siRNA Oligonucleotides". Molecular Pharmaceutics 6.3 (2019): 686-695.

\section{Assets from publication with us}

- Prompt Acknowledgement after receiving the article

- Thorough Double blinded peer review

- Rapid Publication

- Issue of Publication Certificate

- High visibility of your Published work

Website: https://www.actascientific.com/

Submit Article: https://www.actascientific.com/submission.php

Email us: editor@actascientific.com

Contact us: +919182824667 